Cite this: RSC Adv., 2017, 7, 6411

Received 12th December 2016

DOI: $10.1039 / c 6 r a 28110 a$

www.rsc.org/advances

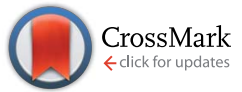
Accepted 9th January 2017

\section{Photophysical properties of betaxanthins: miraxanthin V - insight into the excited-state deactivation mechanism from experiment and computations $\dagger$}

\author{
Stanistaw Niziński, ${ }^{a}$ Monika Wendel, ${ }^{a}$ Michał F. Rode, ${ }^{\text {tb }}$ Dorota Prukała, ${ }^{c}$ \\ Marek Sikorski, ${ }^{c}$ Stawomir Wybraniec ${ }^{d}$ and Gotard Burdziński*a
}

\begin{abstract}
Miraxanthin V is a betaxanthin dye occurring in Caryophyllales plants. This work describes its photophysical properties in aqueous and alcoholic solutions. After excitation at $\lambda_{\text {exc }}=480 \mathrm{~nm}(2.6 \mathrm{eV})$, transient absorption spectrum of the excited $S_{1}$ state was observed $\left(S_{1} \rightarrow S_{n}\right.$ absorption band at $\left.\lambda_{\text {probe }}=394 \mathrm{~nm}\right)$. The $S_{1}$ state population decays with two time constants ( 4.2 ps and 24.2 ps in water) corresponding to the photoexcited miraxanthin $V$ in its two major stereoisomeric forms. The $S_{1}$ state decay is mainly radiationless, since the fluorescence quantum yield is low $\left(\Phi_{\mathrm{F}}=0.003\right.$ in water, the smallest value among all of the studied betaxanthins). Strong correlations were obtained between the solvent viscosity and the $\mathrm{S}_{1}$ state lifetime in linear alcohols, as well as in methanolic solutions at low temperatures. These correlations suggest that intramolecular rotation or conformational relaxation precedes the $S_{1}$ state decay. Full recovery of the electronic ground state $S_{0}$ was observed after excitations at $\lambda_{\text {exc }}=480 \mathrm{~nm}$ and $298 \mathrm{~nm}(4.2 \mathrm{eV})$, without forming the triplet $T_{1}$ state or any photoproducts. Experimental results are complemented by the $a b$ initio $S_{0}$ state calculations using MP2 and DFT methods, and also by the excited-state calculations at the $A D C(2)$ and TD-DFT levels of theory. The ab initio $A D C(2)$ results give insight into the mechanism of the excited-state deactivation through the conical intersection region. We conclude that most probably the excited-state deactivation process is initiated by the rotation about the $\mathrm{C}=\mathrm{N}$ double bond, present in the chain linking the electron-donor catechol to the electron-acceptor dihydropyridine group, and it is followed by collective geometry changes involving a large rotation about the $\mathrm{C}=\mathrm{C}$ double bond. This mechanism seems to be universal for different betaxanthin dyes present in plants, and plays a photoprotective role.
\end{abstract}

\section{Introduction}

Betaxanthins are yellow dyes present in Caryophyllales plants. ${ }^{1}$ Their photophysical properties, such as efficient light to heat conversion, indicate that betaxanthins play a photoprotective role in biological systems. ${ }^{2,3}$ Recent studies for betaxanthins in solutions show that the major $S_{1}$ state deactivation path is due to the $\mathrm{S}_{1} \rightarrow \mathrm{S}_{0}$ internal conversion, while fluorescence contributes only

\footnotetext{
${ }^{a}$ Quantum Electronics Laboratory, Faculty of Physics, Adam Mickiewicz University in Poznań, Umultowska 85, Poznań, 61-614, Poland. E-mail: gotardb@amu.edu.pl

${ }^{b}$ Institute of Physics, Polish Academy of Sciences, Aleja Lotników 32/46, 02-668 Warsaw, Poland. E-mail: mrode@ifpan.edu.pl

${ }^{c}$ Faculty of Chemistry, Adam Mickiewicz University in Poznań, Umultowska 89b, 61614 Poznań, Poland

${ }^{d}$ Faculty of Chemical Engineering and Technology, Institute C-1, Section of Analytical Chemistry, Cracow University of Technology, Warszawska 24, 31-155 Cracow, Poland $\dagger$ Electronic supplementary information (ESI) available: Results of stationary and time-resolved spectroscopic measurements, the results of quantum chemical calculations. See DOI: 10.1039/c6ra28110a
}

weakly $\left(\Phi_{\mathrm{F}}<0.01\right.$ in $\left.\mathrm{H}_{2} \mathrm{O}\right)$, with neither the $\mathrm{S}_{1} \rightarrow \mathrm{T}_{1}$ intersystem crossing nor the product formation ever observed. ${ }^{2,3}$ The internal conversion is likely enhanced by the intramolecular rotations, since an increase in the solvent viscosity causes an increase of the $\mathrm{S}_{1}$ state lifetime and fluorescence quantum yield. Advanced quantum chemical calculations are required for a better understanding of the mechanisms responsible for the efficient internal conversion. Presently, we use miraxanthin V (MIR, Fig. 1) as a model betaxanthin dye, which probably has the highest rate of the internal conversion among betaxanthins, as follows from its lowest fluorescence intensity. ${ }^{4}$

Stintzing et al. have characterized MIR structure using ${ }^{13} \mathrm{C}-\mathrm{NMR}$ spectroscopy. ${ }^{5}$ Four stereoisomeric forms have been identified, existing in a 49:36:8:7 equilibrium mixture in slightly acidified aqueous solutions. Gandía-Herrero et al. have developed a protocol for semi-synthesis of MIR by condensation of betalamic acid with dopamine, followed by HPLC purification. ${ }^{6}$ MIR undergoes thermal degradation in solutions, being sufficiently stable for spectroscopic studies at room temperature. 

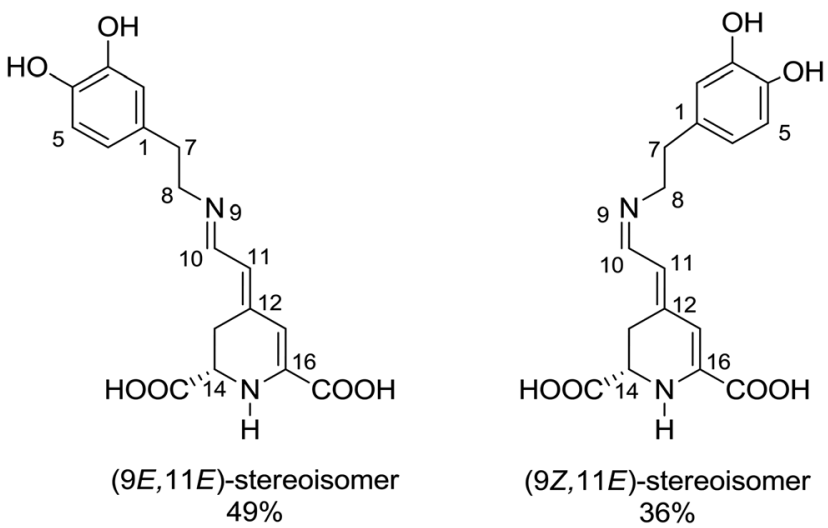

Fig. 1 The two dominant stereoisomers of miraxanthin $\mathrm{V}$ in aqueous solutions. ${ }^{5}$

It is more stable at low temperatures in the $\mathrm{pH}$ range between 4 and 7., ${ }^{7,8}$ The absorption maximum of MIR in water is at about $472 \mathrm{~nm}(2.63 \mathrm{eV}),{ }^{9,10}$ while its fluorescence spectrum peaks at $512 \mathrm{~nm}(2.42 \mathrm{eV}){ }^{4}$ The MIR molar absorption coefficient is expected to be similar to those of other betaxanthins, $\varepsilon_{\max }=48000$ $\mathbf{M}^{-1} \mathrm{~cm}^{-1} .2,3$ MIR is the dominant dye in yellow Mirabilis jalapa L., Portulaca oleracea L. and Celosia argentea L. inflorescences, ${ }^{\mathbf{1 1}-13}$ with flower fluorescence patterns presumably attracting insects pollinators. ${ }^{13,14}$ MIR has a potential to be used as food colorant. ${ }^{12,15}$ It might also be used in DSSC (Dye Sensitized Solar Cells), ${ }^{16}$ where we need to understand the $S_{1}$ state intramolecular deactivation (the undesirable phenomenon) competing with the electron injection (the desirable phenomenon).

The aim of this work is characterization of the MIR photophysical properties using stationary and time-resolved optical spectroscopies. The solvent viscosity is probably an important factor controlling the $S_{1}$ state deactivation dynamics that will be explored. Theoretical studies will elucidate the mechanistic reasons behind the efficient radiationless $S_{1} \rightarrow S_{0}$ transition, by calculating the excited-state minimum-energy path for the isomerization reaction. The new insights are expected to be important for betaxanthins, and also for betacyanins in general.

\section{Results and discussion}

\section{Stationary absorption and fluorescence spectra}

The recorded absorption and fluorescence spectra $\left(\lambda_{\text {exc }}=\right.$ $470 \mathrm{~nm}$ ) of MIR in aqueous and alcoholic solutions are presented in Fig. 2. The absorption band maximum is weakly affected by the solvent, being located around $\lambda_{\mathrm{abs}}^{\max }=475 \mathrm{~nm}$.

These spectra correspond to the $\mathrm{S}_{0} \rightarrow \mathrm{S}_{1}$ transition, predicted using TD-DFT calculations (see Table $\mathrm{S} 1$ in ESI $\dagger$ ). The shapes of MIR absorption spectra are all similar in alcoholic solutions, differing slightly in water. This is also confirmed by calculations that predict no significant difference between the absorption spectra of MIR in $\mathrm{H}_{2} \mathrm{O} v s$. $\mathrm{MeOH}$ (see Table S1 $\dagger$ ). However, the fluorescence maxima $\lambda_{\mathrm{f}}^{\max }$ show a stronger dependence on solvent. The emission spectra in ethylene glycol and water are red-shifted $\left(\lambda_{\mathrm{f}}^{\max } \approx 510 \mathrm{~nm}\right.$, Table 1) in

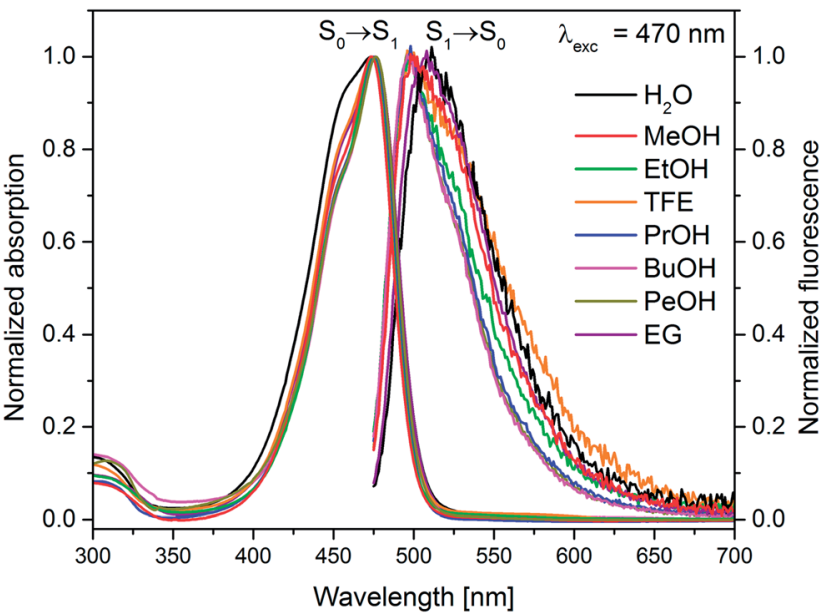

Fig. 2 Normalized stationary absorption and fluorescence spectra of miraxanthin $\mathrm{V}$ in selected solvents.

comparison to linear alcoholic and TFE solutions $\left(\lambda_{\mathrm{f}}^{\max } \approx 498\right.$ $\mathrm{nm})$. The experimental Stokes shift $\left(\Delta \nu_{\mathrm{s}}=\tilde{\nu}_{\mathrm{abs}}^{\max }-\tilde{\nu}_{\mathrm{f}}^{\max }\right)$ reaches the highest value $\left(\approx 1530 \mathrm{~cm}^{-1}\right)$ in water.

The MIR fluorescence excitation spectrum agrees with the absorption spectrum (Fig. S1B $\dagger$ ) implying a similarity of the shape of the absorption spectra of two major MIR stereoisomers in $\mathrm{H}_{2} \mathrm{O}$, in line with calculations (Table $\mathrm{S} 1 \dagger$ ). The contour plot of $3 \mathrm{D}$ fluorescence of MIR in water is shown in Fig. S2. $\dagger$ Fluorescence is placed in one zone at $480-640 \mathrm{~nm}$ in the emission scale. The peak of the contour plot shows a maximum at $\lambda_{\mathrm{f}}^{\max } \approx 510 \mathrm{~nm}$ upon the excitation wavelength $\lambda_{\text {exc }} \approx 470 \mathrm{~nm}$. The shapes of the emission spectra weakly depend on the excitation wavelength. The fluorescence quantum yields $\Phi_{\mathrm{F}}$, determined with rhodamine $6 \mathrm{G}$ as a reference with quantum yield $\Phi_{\mathrm{F}}^{\mathrm{ref}}=0.9,{ }^{17}$ are in the range of $3-22 \times 10^{-3}$ in the solvents tested (Table 1). The clear $\Phi_{\mathrm{F}}$ dependence on the solvent viscosity in linear alcohols (Fig. S3†) agrees with the excited state behavior of other betaxanthins. ${ }^{2,3}$ The smallest $\Phi_{\mathrm{F}}$ value is observed in water $\left(\Phi_{\mathrm{F}}=3 \times 10^{-3}\right)$ which is the lowest value among the betaxanthins studied so far $\left(\Phi_{\mathrm{F}}=5.3\right.$ and $7.3 \times 10^{-3}$ for indicaxanthin and vulgaxanthin $\mathrm{I}$ in $\mathrm{H}_{2} \mathrm{O}$, respectively, ${ }^{2,3}$ see Table $\mathrm{S} 2 \dagger$ ). It also agrees with Gandía-Herrero et al. reporting that the smallest fluorescence intensity is observed for MIR in relation to other betaxanthins. ${ }^{4}$ The weak fluorescence of MIR has been explained by the lack of electron withdrawing group (such as carboxyl) in the amine and the electron-donating character of the catechol moiety. ${ }^{4}$

On the basis of the stationary measurements, we determined the radiative rate constant for MIR in water using the StricklerBerg relation: ${ }^{18}$

$$
k_{\mathrm{r}}=2.88 \frac{10^{-9} n^{2}}{\left\langle\tilde{\nu}_{\mathrm{f}}^{-3}\right\rangle_{\mathrm{Av}}} \int \varepsilon(\tilde{\nu}) \frac{\mathrm{d} \tilde{\nu}}{\tilde{\nu}}
$$

where

$$
\left\langle\tilde{\nu}_{\mathrm{f}}^{-3}\right\rangle_{\mathrm{Av}}=\frac{\int \tilde{\nu}^{-3} F(\tilde{\nu}) \mathrm{d} \tilde{\nu}}{\int F(\tilde{\nu}) \mathrm{d} \tilde{\nu}}
$$


Table 1 Fluorescence quantum yields $\Phi_{\mathrm{F}}\left(\lambda_{\mathrm{exc}}=470 \mathrm{~nm}\right)$, maxima of the $\mathrm{S}_{0} \rightarrow \mathrm{S}_{1}$ absorption bands $\left(\lambda_{\mathrm{abs}}^{\max }\right)$, $\mathrm{S}_{1}$ fluorescence bands $\left(\lambda_{\mathrm{f}}^{\max }\right)$ and Stokes shift values for miraxanthin $\mathrm{V}$ in aqueous and alcoholic solutions

\begin{tabular}{llllll}
\hline Solvent & Viscosity $^{a}[\mathrm{cP}]$ & $\Phi_{\mathrm{F}}\left[\times 10^{-3}\right]^{b}$ & $\lambda_{\mathrm{abs}}^{\max }\left(\mathrm{S}_{0} \rightarrow \mathrm{S}_{1}\right)[\mathrm{nm}]$ & $\lambda_{\mathrm{f}}^{\max }[\mathrm{nm}]$ & Stokes shift $\left[\mathrm{cm}^{-1}\right]$ \\
\hline Water & 0.89 & 3 & 474 & 511 & 1528 \\
Methanol & 0.54 & 4.7 & 474 & 500 & 1097 \\
Ethanol & 1.07 & 7.6 & 475 & 497 & 932 \\
Trifluoroethanol & 1.73 & 5.8 & 475 & 498 & 972 \\
1-Propanol & 1.945 & 14 & 475 & 497 & 928 \\
1-Butanol & 2.54 & 20 & 477 & 497 & 932 \\
1-Pentanol & 3.619 & 15 & 478 & 508 & 844 \\
Ethylene glycol & 16.1 & &
\end{tabular}

here, $\varepsilon(\tilde{\nu})$ is the molar absorption coefficient, $F(\tilde{\nu})$ the fluorescence intensity, $\tilde{v}$ the wavenumber, $n$ the refractive index. ${ }^{18}$ Note, that $F(\tilde{\nu})=\lambda^{2} F(\lambda)$. The resulting calculated value is $k_{\mathrm{r}}=2.2$ $\times 10^{8} \mathrm{~s}^{-1}$ for MIR in water. Note that the $\mathrm{S}_{0} \rightarrow \mathrm{S}_{1}$ absorption band is well-defined, with the molar extinction coefficient $\varepsilon_{\max }$ $\approx 48000 \mathrm{M}^{-1} \mathrm{~cm}^{-1}$.

\section{Transient absorption spectroscopy}

Time-resolved absorption measurements were performed in the spectral range of 330-660 $\mathrm{nm}$ with excitation at $\lambda_{\text {exc }}=480 \mathrm{~nm}$. Fig. 3 shows typical data obtained for MIR in water. Note that two separate contributions are responsible for the negative band: (i) the bleaching at $\approx 470 \mathrm{~nm}$ caused by the reduced MIR concentration in the ground state $S_{0}$ upon the sample photoexcitation (ii) the $\mathrm{S}_{1} \rightarrow \mathrm{S}_{0}$ stimulated emission peaking at $\approx 530 \mathrm{~nm}$. The shape of the latter band may be well approximated by the spectrum of the stimulated emission cross-section obtained from the stationary fluorescence spectrum scaled by the factor of $\lambda^{4}$ (Fig. 3B).

The positive band with the maximum at $394 \mathrm{~nm}(3.14 \mathrm{eV})$ is mainly assigned to the $\mathrm{S}_{1} \rightarrow \mathrm{S}_{n}$ absorption, with quite small $\mathrm{S}_{0}$-bleaching contribution at $394 \mathrm{~nm}$. Normalization of the $\mathrm{S}_{1} \rightarrow \mathrm{S}_{n}$ absorption band shows no spectral shift or band narrowing (Fig. S4†) within our signal-to-noise ratio, thus the contributions from solvation and vibrational cooling are not noticeable. A weak contribution of the solvation was to be expected, based on small Stokes shift values (below $1600 \mathrm{~cm}^{-1}$ ) obtained in the stationary measurements, and a weak dependence of the Stokes shift on the solvent polarity (compare water to the less polar methanol, Table 1).

In order to retrieve the $S_{1}$ state lifetime, band integral kinetics were computed using

$$
\mathrm{BI}=\int_{330}^{670} \Delta A(t, \lambda) \frac{\mathrm{d} \lambda}{\lambda},
$$

where the integration is done over the entire probe spectral range (EBI, 330-670 nm). Note that the usage of the band integral minimizes the potential contributions of the vibrational cooling and solvation effects. ${ }^{19}$ Band integral kinetics were also calculated separately for positive (PBI, 330-430 nm) and negative (NBI, 430-670 $\mathrm{nm}$ ) transient absorption bands.
Satisfactory fits were obtained using a double exponential function (Fig. S5 $\dagger$ ). All of the analyzed band integrals (EBI, PBI and NBI) produce quite similar time constants (Table 2), although the NBI data present a better signal-to-noise ratio.

The two time constants $\left(\tau_{1}=4.2 \mathrm{ps}, \tau_{2}=24.2 \mathrm{ps}\right.$, NBI in water) were tentatively assigned to the $\mathrm{S}_{1}$ state lifetimes of the two major stereoisomers. The ratio of the partial amplitudes with the respective time-constants (0.4 and $0.6, \mathrm{NBI}$ in water) reflects the relative populations of the stereoisomers in the $\mathrm{S}_{1}$ state, which likely coincide with the $\mathrm{S}_{0}$ state equilibrium existing before photoexcitation. Indeed, the ratio $0.42: 0.58$ between the two dominant stereoisomers was estimated for MIR in aqueous solutions using the NMR data (Fig. 1). ${ }^{5}$ Based on their respective abundances, we infer that the $E E$ stereoisomer is associated with the longer time constant, while the $Z E$ stereoisomer with a shorter time constant. Inspection of Table 2 also shows that the relative amplitudes of the two exponential components, describing the band integrals kinetics, depend on solvent. Fig. S6 $†$ shows an increase of the partial amplitude $A_{1}$ with solvent polarity $\pi^{*}$. We can expect, that a more polar solvent stabilizes a more polar stereoisomer. Indeed, theoretical predictions show that the $Z E$ form has a larger dipole moment than $E E$ form (Tables $\mathrm{S} 1$ and $\mathrm{S} 3 \dagger$ ).

The global analysis of the transient absorption data results in time constants ( $\tau_{1}=2.2 \mathrm{ps}, \tau_{2}=20.8 \mathrm{ps}$, in water) similar to those deduced from the band integral kinetics (Table 2); note similar shapes of the two respective decay-associated spectra (Fig. 3C).

The MIR transient absorption data in $\mathrm{H}_{2} \mathrm{O}$ (Fig. 3A) were also analyzed by independent fitting of the individual kinetic traces (Fig. S7 $\dagger$ ). The obtained time constants approximately reproduce the values retrieved in the global analysis (Fig. 3C). The exception is at probe $\lambda=493 \mathrm{~nm}$ where the individual timeconstants are too short. This can be explained by contribution from an intermediate absorption, ${ }^{20}$ indeed species nascent upon $\mathrm{S}_{1} \rightarrow \mathrm{S}_{0}$ deactivation can be structurally unrelaxed and possess a red-shifted absorption band in relation to the relaxed MIR in the $S_{0}$ state.

Time-resolved absorption measurements were also performed for MIR in the range of $825-1380 \mathrm{~nm}$ (the NIR range) with the excitation at $\lambda_{\text {exc }}=485 \mathrm{~nm}$ in water (Fig. S8A $\dagger$ ). The 

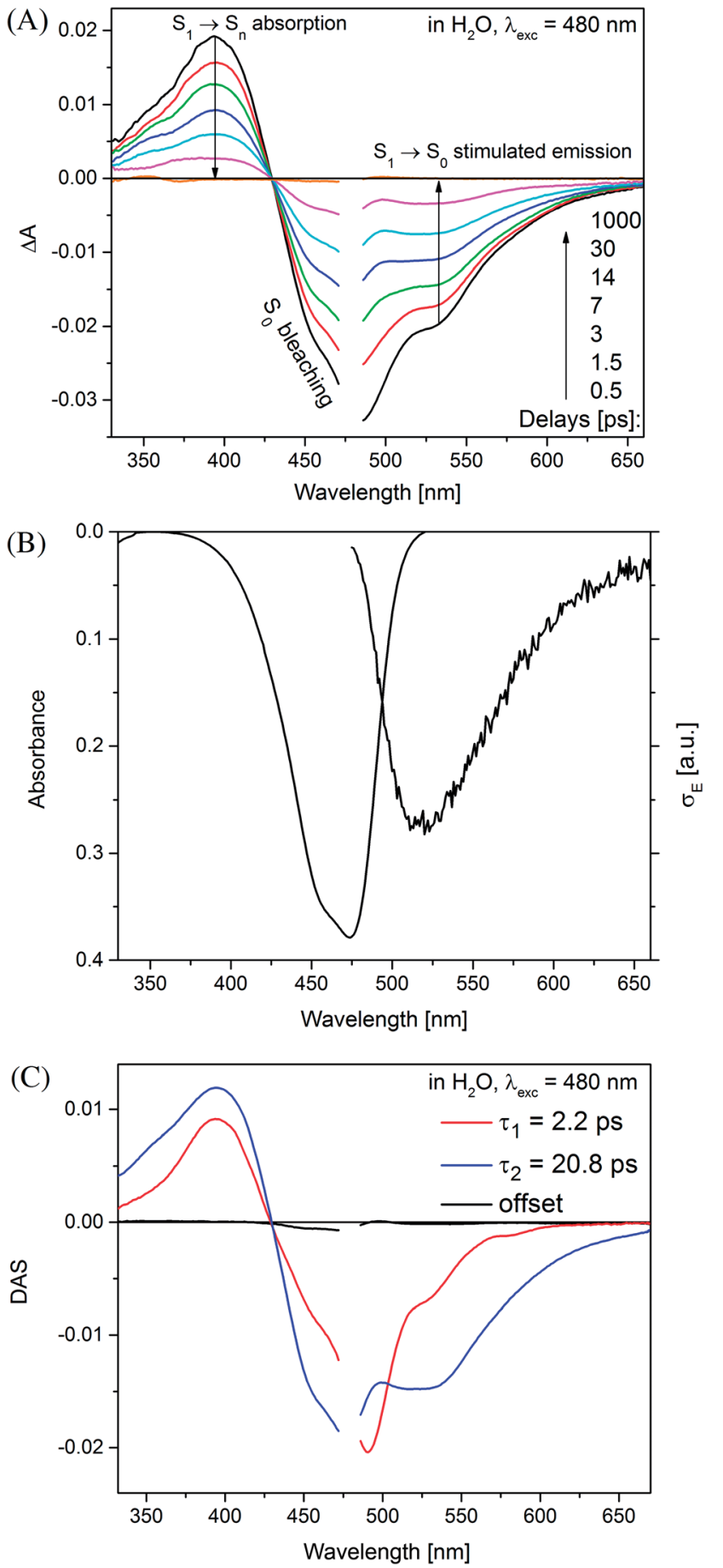

Fig. 3 (A) Transient UV-vis absorption spectra obtained for miraxanthin $V$ in water $\left(\lambda_{\text {exc }}=480 \mathrm{~nm}\right.$ ). (B) Reversed UV-vis absorption and cross-section $\sigma_{\mathrm{E}}$ spectrum for stimulated emission. (C) Decay associated spectra obtained by the two-exponential fit.

recorded $\mathrm{S}_{1} \rightarrow \mathrm{S}_{m}(m>1)$ absorption band is located around $\approx 920 \mathrm{~nm}$. The band integral over the entire spectral range (825$1380 \mathrm{~nm}$ ) was calculated. The two time constants obtained from the double exponential fit: $2.7 \mathrm{ps}(0.41)$ and $21.9 \mathrm{ps}(0.59)$, are in a reasonable agreement with those derived from the global analysis (Fig. $\mathrm{S} 8 \mathrm{~B} \dagger$ ). Thus, the determined amplitude associated spectra (Fig. $\mathrm{S} 8 \mathrm{~B} \dagger_{\dagger}$ ) likely reflect respective stereoisomers $\mathrm{S}_{1}$ state absorption bands. Similar transient absorption data are produced for MIR in $\mathrm{MeOH}$ (Fig. S9A $\dagger$ ), with the amplitude associated spectra resembling those in $\mathrm{H}_{2} \mathrm{O}$.

The long-lived stereoisomer in the $\mathrm{S}_{1}$ state mainly contributes to the stationary fluorescence spectra and to the fluorescence quantum yield $\Phi_{\mathrm{F}}$. This also agrees with inspection of the strength of the stimulated emission transient bands at longer delay times exceeding $6 \mathrm{ps}$, Fig. 3A. The radiative rate constant for this stereoisomer was calculated using the standard expression:

$$
k_{\mathrm{r}} \tau_{2}=\Phi_{\mathrm{F}}
$$

where $\Phi_{\mathrm{F}}$ is fluorescence quantum yield, and $\tau_{2}$ is the lifetime of the long-lived stereoisomer. Linear regression was performed (Fig. S10 $\dagger$ ) and the radiative rate constant of MIR $k_{\mathrm{r}}$ was determined at $k_{\mathrm{r}}=2 \times 10^{8} \mathrm{~s}^{-1}$. Note that $k_{\mathrm{r}}$ agrees with value $2.2 \times$ $10^{8} \mathrm{~s}^{-1}$ calculated from the Strickler-Berg relation (1) for MIR in water.

Very similar spectral shape of transient absorption data (Fig. S11A $\dagger$ ) at delays longer than 3 ps was obtained for MIR in water using excitation at $\lambda_{\text {exc }}=298 \mathrm{~nm}$ instead of $480 \mathrm{~nm}$ (Fig. 3A). The decay time-constants retrieved from band integral analysis (NBI, $\tau_{1}=3.8 \mathrm{ps}, \tau_{2}=23.6 \mathrm{ps}$ ) are comparable to those obtained with $\lambda_{\text {exc }}=480 \mathrm{~nm}$. Inspection of transient absorption kinetics at probe wavelengths $460 \mathrm{~nm}$ and $520 \mathrm{~nm}$ (Fig. S11E $\dagger$ ) shows an extra decay $(460 \mathrm{~nm})$ and rise $(520 \mathrm{~nm})$ with a timeconstant of 0.6 ps. This time-constant likely reflects vibrationally hot population of the $S_{1}$ state generated through the $\mathrm{S}_{n} \rightarrow \mathrm{S}_{1}$ internal conversion process, following the initial $\mathrm{S}_{0} \rightarrow \mathrm{S}_{n}(n>1)$ state photoexcitation (with $\lambda_{\mathrm{exc}}=298 \mathrm{~nm}$ ). Thus, the vibrationally hot $S_{1}$ state contributes to the fast decay of the stimulated emission at probe $460 \mathrm{~nm}$. In contrast, probe $520 \mathrm{~nm}$ shows the initial growth $(0.6 \mathrm{ps})$ of the stimulated emission signal because the vibrationally relaxed $\mathrm{S}_{1}$ state population increases. The early changes caused by the $\mathrm{S}_{1}$ state vibrational relaxation are virtually absent with a long excitation wavelength $\left(\lambda_{\text {exc }}=480 \mathrm{~nm}\right.$, Fig. S11E $\left.\dagger\right)$, since under these conditions a small excess of vibrational energy is produced in the $S_{1}$ state. A very fast heat dissipation capability of MIR can be explained by strong solute-solvent coupling through hydrogen bonding. Note that the vibrational cooling in case of betanin has been also described by a short time-constant $(\approx 0.9 \mathrm{ps}$ in $\left.\mathrm{H}_{2} \mathrm{O}\right) .{ }^{21}$

The transient absorption measurements were also performed for MIR in alcoholic solutions with the excitation at $\lambda=$ $480 \mathrm{~nm}$ (Fig. S12†). The time constants deduced from both global and band integral analysis all depend on the solvent viscosity in linear alcohols (Table 2). Note that while the dynamics is affected by the solvent, the shape of the transient absorption spectra remains unchanged (Fig. S12†). Additional measurements were performed at temperatures between 180 and $300 \mathrm{~K}$ for MIR methanolic solutions, and again a correlation of the $\mathrm{S}_{1}$ lifetime $v s$. viscosity $\eta$ was observed, although the influence of temperature should also be considered (Fig. S13, data in Table S4†). These viscosity-dependent data may be described by a commonly used empirical model. ${ }^{22}$ Thus, the 
Table 2 Miraxanthin $\vee S_{1}$ state decay time constants retrieved from the band integrals and the global analysis of the transient UV-vis data ${ }^{a}$

\begin{tabular}{|c|c|c|c|c|c|c|c|}
\hline & Viscosity $[\mathrm{cP}]$ & $\pi^{*}$ & \multicolumn{2}{|l|}{ Positive BI } & \multicolumn{2}{|c|}{ Negative BI } & Global analysis $\tau_{1}, \tau_{2}^{c}[\mathrm{ps}]$ \\
\hline Methanol & 0.54 & 0.60 & $0.34,0.66$ & $6.1,33.3$ & $0.35,0.65$ & $6.9,36.1$ & $5.6,35.9$ \\
\hline Ethanol & 1.07 & 0.54 & $0.23,0.77$ & $5.6,56.7$ & $0.24,0.76$ & $6,58.4$ & $5.7,58.9$ \\
\hline Trifluoroethanol & 1.73 & 0.73 & $0.32,0.68$ & $5.3,40.1$ & $0.34,0.66$ & $6.1,43.4$ & $4.5,40.7$ \\
\hline 1-Pentanol & 3.619 & 0.40 & $0.27,0.73$ & $8.1,121.4$ & $0.27,0.73$ & $10.4,127.8$ & $7.7,126.7$ \\
\hline Ethylene glycol & 16.1 & 0.92 & $0.36,0.64$ & $9.6,82.2$ & $0.37,0.63$ & $9.5,83.2$ & $7.6,79$ \\
\hline
\end{tabular}

rates of the $S_{1}$ decay in methanol as a function of temperature, and also in linear alcohols at $T=22{ }^{\circ} \mathrm{C}$, may all be fitted using the following equation: ${ }^{23-26}$

$$
k=\frac{\nu}{\eta^{\alpha}} \exp \left(-\frac{E_{\mathrm{A}}}{R T}\right)
$$

where $\eta$ is the solvent viscosity, $T$ temperature, $R$ the ideal gas constant, $k$ the reciprocal of the $\mathrm{S}_{1}$ state lifetime. Here $\alpha$ indicates the influence of viscosity (dynamical molecule-solvent friction) upon the overall $\mathrm{S}_{1}$ state relaxation, with $0 \leq \alpha \leq 1, E_{\mathrm{A}}$ is the activation energy of the barrier between the locally excited state and the twisted state, $\nu$ describes the pressure exerted by MIR at unit viscosity. After applying the logarithm, we obtain:

$$
\ln (k)=\ln (\nu)-\alpha \ln (\eta)-\frac{E_{\mathrm{A}}}{R T}
$$

The fitted surface is presented in Fig. S14. $\uparrow$ The parameter values $\alpha_{2}=0.74 \pm 0.04, E_{\mathrm{A} 2}=57 \pm 33 \mathrm{~cm}^{-1}$, and $\alpha_{1}=0.25 \pm$ $0.10, E_{\mathrm{A} 1}=350 \pm 80 \mathrm{~cm}^{-1}$ were obtained for the long- and shortlived stereoisomers, respectively. As expected, ${ }^{26}$ the higher barrier $\left(E_{\mathrm{A} 1}>E_{\mathrm{A} 2}\right)$ for the internal rotations or conformational relaxation in the singlet excited state should be accompanied by a weaker dependence of the rate $k$ on viscosity $\left(\alpha_{1}<\alpha_{2}\right)$. The activation energy for both stereoisomers is small and comparable to $k T$ value in room temperature $\left(\approx 210 \mathrm{~cm}^{-1}\right)$, thus the solvent viscosity is the main factor controlling the $S_{1}$ state lifetime. Comparing the $\alpha$ values $\left(\alpha_{2}=0.74\right.$ vs. $\left.\alpha_{1}=0.25\right)$, we deduce that the stereoisomer $2(E E)$ experiences a stronger friction against the solvent molecules. Indeed, a more hampered rotation around the central bridge bonds is expected for the $E E$ stereoisomer (Fig. 1) due to the increased friction of the dopamine moiety against the solvent molecules.

\section{Quantum chemical calculations}

The NMR data indicate that MIR exists in solution in an equilibrium between the two main stereoisomers: $E E(9 E, 11 E)$ and $Z E(9 Z, 11 E)$, which are the most abundant (see Fig. 1). ${ }^{5}$ The quantum chemical calculations produced the optimized structures of the most abundant stereoisomers shown in Fig. S15. $\dagger$ In both the forms the electron-donating catechol moiety lies in the plane that is approximately parallel to the plane containing the electron-accepting moiety and the $\mathrm{C}_{11}, \mathrm{C}_{10}, \mathrm{~N}_{9}$ and $\mathrm{C}_{8}$ atoms. The two moieties contained in the above-mentioned planes are linked by the $\mathrm{C}_{7}-\mathrm{C}_{8}$ single bond bridge - a linkage of a geometry typical for saturated hydrocarbons. Such molecular arrangement makes the potential energy profile asymmetric with respect to the rotations described in this paragraph. Both the B3LYP/cc-pVDZ and MP2/cc-pVDZ calculations in gas phase confirm that the $Z E$ isomer is by $\approx 0.16 \mathrm{eV}$ higher in energy than the most stable $E E$ form. The calculated excitation energies along with the oscillator strengths for different rotameric forms of MIR (computed with the aid of the TD-DFT method for different solvents and in vacuum) are presented in Tables $\mathrm{S} 1$ and $\mathrm{S} 3, \uparrow$ with $\mathrm{ADC}(2)$ results given for comparison. The $\mathrm{S}_{0} \rightarrow \mathrm{S}_{1}$ transition was computed at $401 \mathrm{~nm}(3.09 \mathrm{eV})$ with oscillator strength $f=0.20$ for $E E$, and at $422 \mathrm{~nm}(2.94 \mathrm{eV})$ with lower intensity for $Z E$ in vacuum (Table $\mathrm{S} 1 \dagger$ ). This band gets significantly stronger for both forms in polar solvents (water and alcohols), as its oscillator strength $f$ increases to $\approx 0.6$ (see Table S1 $\dagger$ ). Moreover, the calculations show that the two absorption peaks for both the $E E$ and $Z E$ forms are even closer in a given solvent (alcohol or water) than in vacuum. This computational result suggests that the photoexcitation at $\lambda_{\mathrm{exc}} \approx$ $480 \mathrm{~nm}$ in a given solvent results in an initial population of the isomers in the $S_{1}$ state that reproduces the equilibrium existing in the $S_{0}$ state. The molecular orbitals involved in the $S_{0} \rightarrow S_{1}$ transition were plotted with isosurfaces at 0.03 value (Table $\mathrm{S} 5 \dagger)$. The major contribution to this transition is provided by $\operatorname{HOMO}(\pi) \rightarrow$ LUMO $\left(\pi^{*}\right)$ component, while the second most relevant HOMO-1 $(\pi) \rightarrow$ LUMO $\left(\pi^{*}\right)$ contribution is a little smaller for both the forms. The coefficients of electronic configurations contributing to the $\operatorname{ADC}(2) S_{1}$ state wavefunction are given in Table S5. $\dagger$ Note that the $S_{0} \rightarrow S_{1}$ transition has a $\pi, \pi^{*}$ character for both the $E E$ and $Z E$ forms. The shape of orbitals indicates that after excitation within the Franck-Condon area in the $S_{1}$ state, a part of the electron density moves from the electron-donating catechol moiety to the one of the electron-accepting carboxyl groups, ${ }^{27}$ suggesting that MIR may act as a sensitizer in DSSC. The presence of $\mathrm{COOH}$ groups in MIR facilitates anchoring of this dye to the $\mathrm{TiO}_{2}$ layer. ${ }^{28}$ The difference between the experimental excitation wavelength (474 
Table 3 Comparison of the geometric parameters (bond lengths, in $\AA$ ), adiabatic energies $\left(E_{a}\right.$, in eV) and dipole moments $(\mu$, in debye) for the equilibrium ground state $\left(S_{0}\right)$ and the excited state $\left(S_{1}\right)$ geometries optimized at the MP2/cc-pVDZ (for $S_{0}$ ) and the ADC2/cc-pVDZ (for $S_{1}$ ) levels of theory. The fluorescence energy $\left(E_{f}\right.$, in $\left.\mathrm{eV}\right)$ and oscillator strength $f\left(\mathrm{~S}_{1} \rightarrow \mathrm{S}_{0}\right)$ are given for the $\mathrm{S}_{1}$-state

\begin{tabular}{|c|c|c|c|c|c|c|c|c|}
\hline & $\mathrm{C}_{8}-\mathrm{N}_{9}$ & $\mathrm{C}_{10}=\mathrm{N}_{9}$ & $\mathrm{C}_{10}-\mathrm{C}_{11}$ & $\mathrm{C}_{11}=\mathrm{C}_{12}$ & $E_{\mathrm{a}}[\mathrm{eV}]$ & $E_{\mathrm{f}}[\mathrm{eV}]$ & $\mu[\mathrm{D}]$ & $f\left(\mathrm{~S}_{1} \rightarrow \mathrm{S}_{0}\right)$ \\
\hline $\mathrm{S}_{0} E E$ & 1.455 & 1.299 & 1.454 & 1.374 & 0.00 & & 2.8 & \\
\hline $\mathrm{S}_{1} E E \pi \pi^{*}$ & 1.447 & 1.318 & 1.427 & 1.415 & 3.32 & 2.98 & 5.1 & 0.541 \\
\hline $\mathrm{S}_{1} Z E \pi \pi^{*}$ & 1.455 & 1.321 & 1.429 & 1.420 & 3.38 & 2.96 & 5.3 & 0.512 \\
\hline $\mathrm{S}_{0} Z E$ & 1.457 & 1.302 & 1.460 & 1.377 & 0.16 & & 3.4 & \\
\hline
\end{tabular}

$\mathrm{nm})$ and its predicted theoretical value $(401 \mathrm{~nm})$ is quite significant $\left(3837 \mathrm{~cm}^{-1}=0.48 \mathrm{eV}\right)$, while still at the level of the mean absolute error for the B3LYP functional $(\approx 0.34 \mathrm{eV}$ for excited singlet states). ${ }^{29}$ Note that our attempt to use either a CAM-B3LYP functional or a larger aug-cc-pVTZ basis set with B3LYP functional produced no improvements to the TD-DFT results.

The strongly absorbing $\pi \pi^{*}$ state is always the lowest excited state in the spectrum. Thus, we presume that this is the initially photoexcited state, active in the photophysics of MIR, however, the second lowest excited state has $\mathrm{n} \pi^{*}$ character and lies approx. $0.5 \mathrm{eV}$ above the $\mathrm{S}_{1}\left(\pi \pi^{*}\right)$ state, according to the ADC(2)/ cc-pVDZ method (see Table S3†).

As already mentioned, we expect that upon UV excitation of the $\mathrm{S}_{0} \rightarrow \mathrm{S}_{1}\left(\pi \pi^{*}\right)$ transition, the initially formed $\mathrm{S}_{1}\left(\pi \pi^{*}\right)$ state in solution exists in two major forms: $\mathrm{S}_{1}(E E)$ and $\mathrm{S}_{1}(Z E)$, with the population ratio similar to that of the $\mathrm{S}_{0}$ state. Moreover, the transient absorption data show two characteristic time constants assigned to the $S_{1}$ state lifetimes. Those two $S_{1}\left(\pi \pi^{*}\right)$ state forms are almost isoenergetic in the gas phase, according to the ADC(2)/cc-pVDZ results (compare the adiabatic energies of the ${ }^{1} \pi \pi^{*}$-excited states in Table 3). The electronic structure analysis indicates locally-excited character of the respective minima (see Table S5 $\dagger$ ). Both the electron-donating and accepting orbitals are localized on the dihydropyridine moiety of MIR. However, the experimentally determined $S_{1}$ state lifetimes (4.2 ps and $24.2 \mathrm{ps}$ in water) are relatively short. This suggests existence of an efficient molecular mechanism for the radiationless decay of the electronic excitation. A possible explanation for the fast excited-state deactivation is given by the existence of a low-lying $\mathrm{S}_{1}-\mathrm{S}_{0}$ conical intersection (CI) seam (see the colored beige rectangles in Fig. 4 and bottom-right circle in Fig. 5), where non-adiabatic transition takes place from the excited state $\left(\mathrm{S}_{1}\right)$ to the $\left(\mathrm{S}_{0}\right)$ electronic ground state..$^{30,31}$

Such transitions are the most effective in the geometries where the excited and the ground states become degenerate. In our experiments the dependence of the MIR-S $\mathrm{S}_{1}$ state lifetimes on the solvent viscosity was determined (Fig. S14 $\dagger$ ). This implies that the driving coordinate for the excited-state decay should be a large-amplitude motion described as a rotation about one of the torsional angles. Studying the MIR chemical structure, we may consider several potential reaction coordinates that may drive the system towards the $\mathrm{S}_{1}-\mathrm{S}_{0}$ CI region.
There are six torsional angles which should be considered as a driving coordinate. These are two double bonds: $\mathrm{C}_{11}=\mathrm{C}_{12}$ and $\mathrm{C}_{10}=\mathrm{N}_{9}$, shortly referred to as $\mathrm{C}=\mathrm{C}$ and $\mathrm{C}=\mathrm{N}$, and four single bonds: $\mathrm{C}_{1}-\mathrm{C}_{7}, \mathrm{C}_{7}-\mathrm{C}_{8}, \mathrm{C}_{8}-\mathrm{N}_{9}$, and $\mathrm{C}_{10}-\mathrm{C}_{11}$. Single bonds can be immediately excluded from consideration for a following reason; the shape of the minimum energy profile (MEP) of the excited state calculated along the coordinate described as a rotation about a single bond is known to be parallel to the MEP along the same coordinate for the $\mathrm{S}_{0}$ state,,$^{30,32-34}$ thus such rotation would demand overpassing the excited-state energy barrier as it also exists in the $\mathrm{S}_{0}$ state. This implies that single bond rotations cannot lead to the fast deactivation pathway through the CI regions. We are pointing to the conclusion that rotations about double bonds are good candidates for the driving coordinates for fast deactivation process ongoing in the excited state. Why is that?

There are several known classes of molecules where such excited-state rotations around different type of double bonds

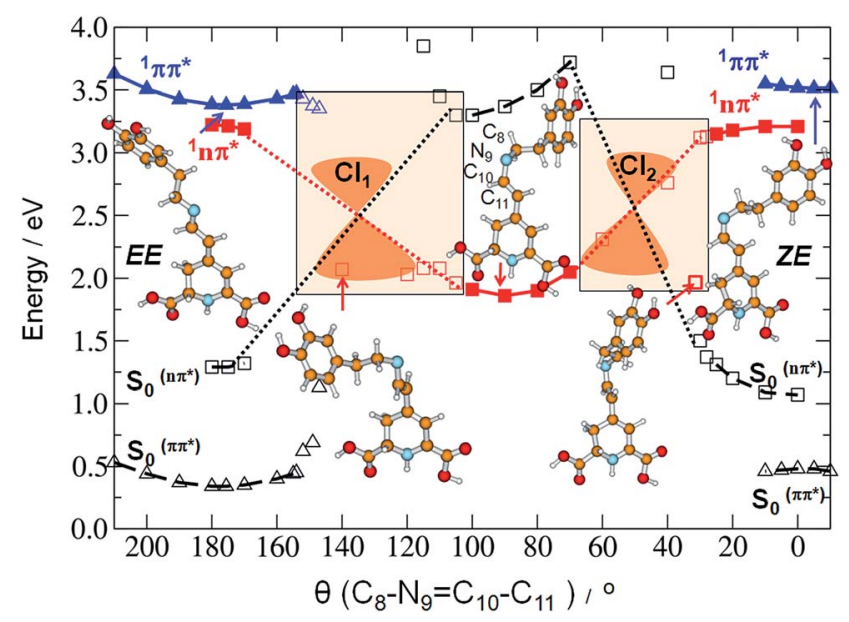

Fig. 4 Potential-energy profiles of miraxanthin $\mathrm{V}$ in the excited states: ${ }^{1} \pi \pi *$ state (blue triangles), ${ }^{1} \mathrm{n} \pi *$ state (red squares) determined at the ADC(2)/def-SV(P) level (MP2/def-SV(P) for the ground state) along the minimum energy path (solid line) for the isomerization between the $E E$ and $Z E$ stereoisomers. $\mathrm{S}_{0}^{\left(\pi \pi^{*}\right)}$ (empty black triangles) and $\mathrm{S}_{0}^{\left(\mathrm{n} \pi^{*}\right)}$ (empty black squares) denote the energy of the ground state calculated along the minimum-energy path of the appropriate excited state (dashed curves). Shaded rectangles denote areas where the ADC(2) iteration cycle ceases to converge due to degeneracy of the $S_{1}$ and the $S_{0}$ states. Empty blue triangles and empty red squares denote the points corresponding to the given ${ }^{1} \pi \pi^{*}$ or ${ }^{1} \mathrm{n} \pi *$ excited state energy profile for which the ADC(2) method ceased to converge. 
(A)

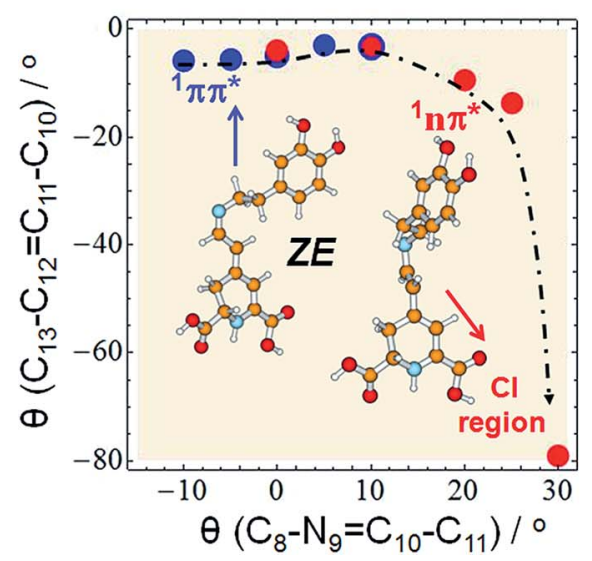

(B)

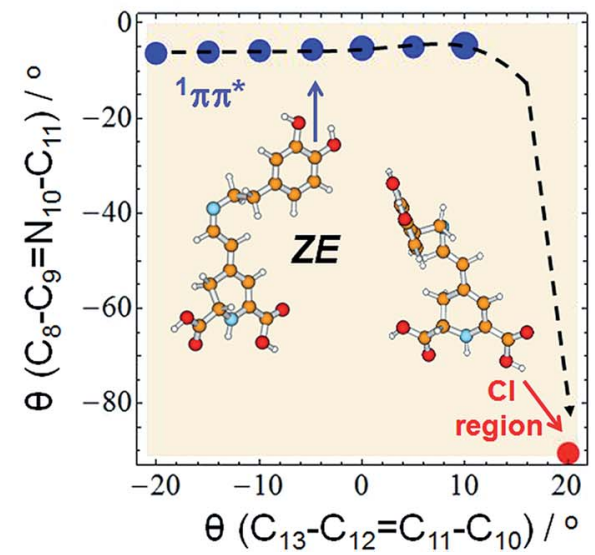

Fig. 5 Minimum-energy profile of the ${ }^{1} \pi \pi^{*}$ (blue circles) and ${ }^{1} n \pi^{*}$ (red circles) excited state of miraxanthin plotted as a function of $\theta\left(C_{8}-N_{9}=C_{10}-C_{11}\right)$ and $\theta\left(C_{13}-C_{12}=C_{11}-C_{10}\right)$ coordinates illustrating the deactivation pathway of the $Z E$ form according to two alternative scenarios: (A) the initial rotation about the $\mathrm{C}=\mathrm{N}$ bond (as in Fig. 4 ) is followed by strong twisting of the $\mathrm{C}=\mathrm{C}$ bond, (B) the initial rotation about the $\mathrm{C}=\mathrm{C}$ bond (as in Fig. S16 $\dagger$ ) followed by large twisting of the $\mathrm{C}=\mathrm{N}$ bond to enter the $\mathrm{Cl}$ region. The red circle in the bottom right corner of the surfaces represents the conical intersection region: $\mathrm{Cl}\left(\mathrm{S}_{0}-\mathrm{S}_{1}\right)$ of a so-called "collapsed" structure. The results were obtained with the aid of the ADC(2)/def-SV(P) method.

are observed, such as azobenzenes where rotation around the $\mathrm{N}=\mathrm{N}$ double bond leads to profound conformational changes. $^{35-38}$ The excited-state rotation around the $\mathrm{C}=\mathrm{C}$ double bond occurs in the extensively studied stilbenes $(Z / E$ isomerization), ${ }^{39,40}$ cyanines, ${ }^{\mathbf{4 1 - 4 3}}$ the 3 -hydroxy-picolinic acid, ${ }^{\mathbf{4 4 , 4 5}}$ and the $\mathbf{7} \mathbf{H Q}^{\mathbf{4 6 , 4 7}}$ molecule. Quite recently, a new photoswitching molecule $N$-(3-pyridinyl)-2-pyridinecarboxamide (NPPCA) has been investigated, where excited state rotation occurs about the $\mathrm{C}=\mathrm{N}$ double bond, ${ }^{48}$ present also in the MIR chromophore; the same effect was observed in light-switchable peptides. ${ }^{33,39}$

Upon UV excitation, a double bond nominally short in the $\mathrm{S}_{0}$ state, may get weaker and longer in the excited state. This effect was detected in a series of derivatives of 7-hydroxy-quinoline $(7 \mathbf{H Q})^{47}$ and salicylidene methylamine. ${ }^{49}$ Thus, a hindered rotation around a nominally double bond in the $S_{0}$ state may become allowed in the $S_{1}$-excited state due to lowering of the barrier, explained by this bond becoming a longer and weaker (single) bond in the $\mathrm{S}_{1}$ state.

Indeed, according to the calculations, comparing the ground- and excited-state equilibrium geometries in MIR, both the $\mathrm{C}=\mathrm{C}$ and $\mathrm{C}=\mathrm{N}$ double bonds undergo elongation upon excitation, with the single $\mathrm{C}-\mathrm{C}$ bond simultaneously getting shorter. This is observed for both the $E E$ and $Z E$ rotamers. The respective geometric parameters are compared in Table 3.

Our calculations indicate an elongation of the $\mathrm{C}=\mathrm{C}$ bond by 0.039 and $0.043 \AA$ for the $E E$ and $Z E$ forms, respectively, comparing their equilibrium ground-state geometry to their excited-state equilibrium geometry; similarly, we obtained elongation of the $\mathrm{C}=\mathrm{N}$ bond by $0.019 \AA$ for both forms. To get insight into the mechanism of deactivation process of MIR the excited-state MEPs were calculated along two driving coordinates, separately: rotations about the $\mathrm{C}_{10}=\mathrm{N}_{9}$ and $\mathrm{C}_{11}=\mathrm{C}_{12}$ double bonds. Until the system is in the ${ }^{1} \pi \pi^{*}$-state minimum, both planes including two ring moieties are almost parallel to one another. Comparison of the estimated in calculations excited-state barriers, indicates that in the initially photoexcited $\mathrm{S}_{1}\left(\pi \pi^{*}\right)$ state, the barrier for the rotation about the $\mathrm{C}_{10}=\mathrm{N}_{9}$ bond is lower than analogous barrier for the $\mathrm{C}_{11}=\mathrm{C}_{12}$ and $\mathrm{C}_{10}-\mathrm{C}_{11}$ rotations, for a given stereoisomer, especially for $E E$ ( $c f$. $4, \mathrm{~S} 16$ and $\mathrm{S} 17 \dagger)$. That is why we may assume that it is a rotation about the $\mathrm{C}=\mathrm{N}$ bond that initiates the deactivation process of overpassing the ${ }^{1} \pi \pi^{*}$-state barrier. Such evolution of the system toward the ${ }^{1} \pi \pi^{*}$-state barrier one may observe in Fig. 5A for which the $\mathrm{C}=\mathrm{C}$ bond does not change much.

The initial $\mathrm{C}=\mathrm{C}$ rotation as ${ }^{1} \pi \pi^{*}$-state deactivation path can be considered for $Z E$ stereoisomer (Fig. 5B), since the barrier for the $\mathrm{C}=\mathrm{C}$ rotation is only slightly higher than that for $\mathrm{C}=\mathrm{N}$ rotation. However in both scenarios (Fig. 5A and B) the initial bond rotation along one coordinate is followed by twisting of the second coordinate before reaching the conical intersection point, where MIR deactivates to the ground state.

The conical intersection region is reached upon the initial rotation about the $\mathrm{C}=\mathrm{N}$ double bond of the $Z E$ form by the dihedral angle of $\approx 30^{\circ}$. The most relevant orbitals that contribute to the nonadiabatic transition between the excited and the ground state in the $\mathrm{S}_{1} / \mathrm{S}_{0}$ conical intersection are shown in Table S7. $\dagger$ The most important in this arbitrarily chosen geometry is the $n \pi *$ contribution, while the $\pi \pi^{*}$ contribution is also present. However, based on this contribution set we can't state that the conical intersection is purely of the $n \pi * / S_{0}$ nature. Its character is thus a mixture of the two contributions.

The photophysical deactivation of MIR proceeds as follows. After photoexcitation of the $E E$ form (as well as $Z E$ ), the $S_{1}\left(\pi \pi^{*}\right)$ state is populated. As shown in Fig. 4, the potential-energy profile of the $S_{1}\left(\pi \pi^{*}\right)$ excited state has a small barrier of $0.09 \mathrm{eV}$ for the rotation about the $\mathrm{C}=\mathrm{N}$ bond (see blue curve in the upper-left corner of Fig. 4). The analogous $\mathrm{S}_{1}\left(\pi \pi^{*}\right)$-state barrier for the $Z E$ form is lower, of $0.04 \mathrm{eV}$ (see the blue curve in the upper-right 
corner of Fig. 4). Such a small barrier may be easily overcome in experiments undertaken at room temperature. It is also evident that a rotation by just about $25-30^{\circ}$, either from the $E E$ or from the $Z E \mathrm{~S}_{1}\left(\pi \pi^{*}\right)$-state minimum form, may put the system into a close proximity of the CI region (beige areas). The process is also shown in Fig. 5A which illustrates the deactivation pathway eye-guided along the points presented in the two-coordination space. In this region $\left(\theta(\mathrm{C}=\mathrm{N}) \approx 30^{\circ}\right)$, the $\mathrm{S}_{1}\left(\pi \pi^{*}\right)$ state meets the descending - ${ }^{1} \mathrm{n} \pi^{*}$ state - which was found to be the second excited state in the absorption spectrum both for the $E E$ and $Z E$ forms (Table $\mathrm{S} 3 \dagger$ ), and $\mathrm{S}_{1}\left(\pi \pi^{*}\right)$ populates this ${ }^{1} \mathrm{n} \pi^{*}$ state. This intersection of the $\pi \pi^{*}$ and $n \pi^{*}$ states is illustrated in Fig. $5 \mathrm{~A}$, where at the intersection point the two states have the same driving coordinate values what helps the transition from one state into another because the barrier between the states disappears. In general, however, one may note that the intersection point presented in Fig. 5A is still just an apparent crossing, since the remaining geometry parameters in the two states can differ. As soon as the rotation about the $\mathrm{C}=\mathrm{C}$ bond is allowed, the optimization procedure immediately heads to the "collapsed" structure with the $\mathrm{C}_{13}-\mathrm{C}_{12}=\mathrm{C}_{11}-\mathrm{C}_{10}$ dihedral $\approx 80^{\circ}$ (shown in the bottom right corner of Fig. $5 \mathrm{~A}$ ), which is a structure with $\approx 60^{\circ}$ angle between the two planes of the ring moieties. Upon large amplitude motion, including other degrees of freedom such as rotation about single bonds in the bridge, the energy of the excited state rapidly decreases and collective relaxation immediately drives the system toward the $\mathrm{CI}\left(\mathrm{S}_{1}-\mathrm{S}_{0}\right)$ region where the nonadiabatic transition to the $S_{0}$ state takes place. Unfortunately this leads the $\mathrm{ADC}(2)$ method to cease in convergence.

Exactly the same mechanism of excited-state deactivation was made for the $E E$ and $Z E$ tautomers (compare the second left and the second right structures in Fig. 4). Moreover, since after overpassing the ${ }^{1} \pi \pi^{*}$-state barrier, the system rotates $\approx 80^{\circ}$ about the $\mathrm{C}=\mathrm{C}$ bond, it can be assumed that the system will come back to the same $S_{0}$ state stereoisomer which was photoexcited (aborted isomerization path), instead of proceeding through the higher-in-energy central path connected with lonely rotation about the $\mathrm{C}=\mathrm{N}$ double bond, in which the $\mathrm{C}=\mathrm{C}$ rotation is not present.

The involvement of the $n \pi^{*}$ state in the overall excited-state deactivation process through the conical intersection region(s) in MIR is an interesting idea. Additional evidence in support is given by the fact that the $n \pi *$-state is found driving the same processes in several other molecules, e.g. for the hydrogenbonded azo aromatic molecules, ${ }^{37}$ dyes with the molecular scaffold of 2-hydroxyazobenzene (Sudan I and Orange II), ${ }^{38}$ and for the picolinic acid derivatives. ${ }^{\mathbf{4 4 , 4 5}}$ In the latter case the ${ }^{1} \mathrm{n} \pi^{*}$ state lies below the ${ }^{1} \pi \pi^{*}$ excited state in all of four tautomeric forms studied. Apparently the electronically excited picolinic acid decays non-radiatively through the conical intersections of the ${ }^{1} \mathrm{n} \pi^{*}$ state with the ground state, quenching the fluorescence of all of the tautomeric forms. ${ }^{44}$

\section{Conclusions}

Experimental data show an increase of the MIR $S_{1}$ state lifetimes with an increase of solvent viscosity in linear alcohols. We postulate that torsional relaxation of the $S_{1}\left(\pi, \pi^{*}\right)$ state around the central bonds $\left(\mathrm{N}=\mathrm{C}\right.$ and/or $\mathrm{C}=\mathrm{C}$ ) leads to $\mathrm{S}_{1} \rightarrow \mathrm{S}_{0}$ radiationless relaxation. This rotational motion is slower in a more viscous solvent, therefore the internal conversion rate is limited by the time required to reach the necessary geometry. The $\mathrm{S}_{1} \rightarrow$ $\mathrm{S}_{0}$ radiationless relaxation is the main deactivation channel for the $S_{1}$ state. Fluorescence is a minor relaxation pathway $\left(\Phi_{\mathrm{F}}<\right.$ 0.03 in all of the solvents tested), with a secondary role in the overall $S_{1}$-population deactivation. We obtained no indications of photoproduct or $\mathrm{T}_{1}$ state formation from the $\mathrm{S}_{1}$ state, with the full $S_{0}$ state recovery observed. The $S_{1}$ state population exhibit biexponential decays in solution attributed to the presence of two major stereoisomers in the ground state of MIR. While numerous photophysical properties, including stationary UVvis absorption, fluorescence, spectral locations of the $S_{1}$ state absorption band and the $S_{1}$ deactivation paths make MIR similar to other betaxanthins studied so far (see Table $\mathrm{S} 2 \uparrow$ for the comparison), ${ }^{2,3}$ MIR exhibits exceptionally low fluorescence quantum yield $\Phi_{\mathrm{F}}$, and relatively short $\mathrm{S}_{1}$ state lifetimes. These features are controlled by structural factors, such as the electron-donating character of the catechol moiety, while the more fluorescent betaxanthins (vulgaxanthin I, indicaxanthin) typically possess an electron-withdrawing group (e.g. carboxyl) in the amine, instead of catechol.

$A b$ initio calculations give insight into the mechanism of fast $\mathrm{S}_{1} \rightarrow \mathrm{S}_{0}$ internal conversion in MIR. Easy access to the CI region through the rotation about the $\mathrm{C}=\mathrm{N}$ and $\mathrm{C}=\mathrm{C}$ bonds explains fast radiationless deactivation of the singlet excited state and its short lifetime. The rotation about the $\mathrm{C}=\mathrm{N}$ double bond seems to initiate the excited-state deactivation process, with an energy barrier lower than that for the rotation about the $\mathrm{C}=\mathrm{C}$ bond, and much lower than that for the rotation about the $\mathrm{C}-\mathrm{C}$ single bond in the central bridge of the molecule. However, as soon as the system leaves the initially photoexcited ${ }^{1} \pi \pi^{*}$-state minimum, collective geometry changes, foremost the rotation about the $\mathrm{C}=\mathrm{C}$ double bond, drives the system toward the $\mathrm{CI}$ region and next to the ground state repopulation, closing the photocycle through the aborted isomerization process. The twisting angle of $\approx 30^{\circ}$ is required for the system to reach the CI region, thus it explains found influence of solvent viscosity on $\mathrm{S}_{1}$ lifetimes and fluorescence quantum yields in solution. Since the other betaxanthins studied so far $^{2,3}$ also have two distinct values of the $S_{1}$ state lifetime, we expect them to have a similar $S_{1}$ state deactivation mechanism, with the $S_{1}$ state decay rate controlled by structural factors, location of the CI region, and solvent viscosity.

\section{Experimental section}

Pure MIR was obtained as proposed by Gandía-Herrero et al. ${ }^{6}$ Before the semi-synthesis of MIR, a separation of the synthetic substrate, indicaxanthin (IND), from the orange-yellow fruits of Opuntia ficus-indica L. Mill, was performed as described previously. ${ }^{2}$ During the semi-synthesis, dopamine was added in a 25fold molar excess to an alkaline solution of betalamic acid obtained directly after IND hydrolysis at $\mathrm{pH} 11.4$ through addition of $25 \% \mathrm{NH}_{4} \mathrm{OH}$. After 20 min since the addition of dopamine, 
the solution was acidified to $\mathrm{pH} 6.5$ by formic acid and chromatographically purified by open column chromatography on C18 sorbent (Merck, Darmstadt, Germany). ${ }^{2}$ Typically, the C18 sorbent was activated with $100 \%$ methanol and then rinsed with $1 \%$ aqueous formic acid. The samples were applied to a column rinsed again with $1 \%$ formic acid and betaxanthin was eluted with methanol. The eluates were pooled and preconcentrated under reduced pressure at $25{ }^{\circ} \mathrm{C}$. Concentrated extract was submitted to semipreparative flash chromatography (preparative HPLC system with LC-20AP pumps, UV-VIS SPD-20AV detector and LabSolutions 5.51 operating software, Shimadzu Corp., Japan) on a C18 flash column (Interchim, France) for the separation of MIR. ${ }^{21}$ Further purification of the pigment was performed on an semipreparative HPLC column Luna C18(2) $250 \times 30 \mathrm{~mm}$ i.d., $10 \mu \mathrm{m}$ (Phenomenex, Torrance, CA, USA).$^{50}$

The details of femtosecond UV-vis-NIR transient absorption spectra have been described elsewhere. ${ }^{2}$ The ultrafast laser system consists of a short-pulse titanium-sapphire oscillator (Mai-Tai, Spectra Physics, 70 fs) followed by a high-energy titanium-sapphire regenerative amplifier (Spitfire Ace, Spectra Physics, $100 \mathrm{fs})$. The $800 \mathrm{~nm}$ beam was split into two beams to generate: (1) a pump ( $\lambda_{\text {exc }}=480,485$ or $\left.298 \mathrm{~nm}\right)$ in the optical parametric amplifier (Topas Prime with a NirUVis frequency mixer) and (2) probe pulses - white light continuum in the UVvis range by using a $\mathrm{CaF}_{2}$ plate $(330-660 \mathrm{~nm}$, Ultrafast Systems, Helios) or in the NIR range by using a YAG crystal (830$1390 \mathrm{~nm}$, Ultrafast Systems, Helios). The remaining $800 \mathrm{~nm}$ photons in the probe pulse were filtered out before the sample. The pump pulse energy was typically about $0.5-0.8 \mu \mathrm{J}$. In most of the transient absorption experiments the absorbance was about 0.5 at the excitation wavelength in a $2 \mathrm{~mm}$ optical path quartz cell. Sample solution was stirred by a Teflon-coated bar. The entire set of pump-probe delay positions was repeated at least four times to ensure data reproducibility. The transient absorption data were corrected for chirp of the white light continuum. The instrument response function (IRF) was about $200 \mathrm{fs}$ (FWHM) in the experiments with $480 \mathrm{~nm}$ excitations, and $350 \mathrm{fs}$ (FWHM) with the excitation at $298 \mathrm{~nm}$. The data were analyzed by determination of the band integral kinetics and fitted with a double-exponential function. The convolution with a Gaussian response function was included into the global fitting procedure (ASUFIT program), and satisfactory fits were obtained. Temperature-dependent experiments between 180 and $300 \mathrm{~K}$ were performed in a liquid nitrogen cryostat (Janis VPF-100). The solutions were placed into a cuvette consisting of two parallel plastic windows, separated by $1.5 \mathrm{~mm}$ distance. The temperature was measured by two thermocouples, located over and under the sample.

Fluorescence emission and excitation spectra were recorded on a Horiba Jobin-Yvon-Spex Fluorolog 3-22 spectrofluorometer. Right-angles geometry was used in all of the steady-state emission measurements. The sample emission and excitation spectra were recorded with three replicates at a reduced scanning rate. The experiments were performed on $3.5 \mathrm{~mL}$ of sample solutions contained in a quartz cell $(1 \mathrm{~cm} \times 1 \mathrm{~cm})$ with the MIR absorbance not exceeding 0.1 at the excitation wavelength $(2 \times$ $10^{-6} \mathrm{M}$ in water). The excitation wavelength was set at $\lambda_{\mathrm{exc}}=$
$470 \mathrm{~nm}$ for the fluorescence measurements. Fluorescence spectra were collected with $1 \mathrm{~nm}$ excitation and emission slits, using $0.5 \mathrm{~s}$ integration time. Fluorescence spectra typically were not corrected for contribution of the solvent Raman scattering (Fig. S1A $\dagger$ ).

For the 3D fluorescence spectra, excitation and emission slits were $2 \mathrm{~nm}$; the acquisition interval was $2 \mathrm{~nm}$ and the integration time was $0.2 \mathrm{~s}$. 3D emission spectra were collected between 480 to $675 \mathrm{~nm}$ in the $355-550 \mathrm{~nm}$ range of the excitation wavelengths, spaced by $5 \mathrm{~nm}$ intervals in the excitation domain. Stationary UV-vis absorption spectra were recorded using a Jasco V-550 spectrophotometer. Alcohols and rhodamine 6G were purchased from Aldrich, water was purchased from Fluka. Unless otherwise stated, experiments were performed at room temperature $\left(22{ }^{\circ} \mathrm{C}\right)$.

\section{Computational methods}

To simulate the UV-absorption spectra of MIR, the equilibrium geometries of the molecular system in its closed-shell ground singlet state $\left(\mathrm{S}_{0}\right)$ were determined at the level of density functional theory (DFT) with the use of the of the B3LYP ${ }^{51}$ hybrid correlation-exchange functional in conjunction with the cc$\mathrm{pVDZ}^{52}$ correlation-consistent basis set as implemented in TURBOMOLE software package. ${ }^{53}$ In the calculation of the excitation energies, mimicking the absorption spectra, the basis set was augmented with diffuse functions (aug-cc-pVDZ ${ }^{52}$ ) and single-point TD-DFT calculations were done on the top of the geometries optimized with the cc-pVDZ basis set. Such calculations are supposed to be in good agreement with the experimental UV-absorption spectra. In the next step, the geometry was optimized in the Conductor-like Screening Model $^{54}$ (Cosmo) implemented in TURBOMOLE, to have comparison of the UV-absorption spectra obtained in the solvent and vacuum. Such satisfactory comparison was done in our report on betanin, ${ }^{21}$ based on TD-DFT calculations with the B3LYP functional $^{51}$ with $6-311++\mathrm{G}(3 \mathrm{df}, 3 \mathrm{pd})$ basis set $^{55}$ (the largest Poplestyle basis set) and polarizable continuum model (PCM) for the solvent, ${ }^{56,57}$ as implemented in Gaussian software package. ${ }^{58}$ Thus, here we use the same approach for MIR.

However, the TD-DFT method is also known to fail in prediction of the excited states with strong charge-transfer, ${ }^{\mathbf{5 9 , 6 0}}$ with small overlap of the two orbitals contributing to the electron excitation (e.g. HOMO and LUMO). To bypass this problem in predicting the excited-state potential energy profile (PE), additional calculations were performed, where the equilibrium geometry of the molecular system in its closed-shell singlet ground state $\left(\mathrm{S}_{0}\right)$ was determined with the MP2 method. ${ }^{61}$ Excitation energies, equilibrium geometries, and response properties of the lowest singlet excited states were calculated using the algebraic diagrammatic construction method of the second order, $\operatorname{ADC}(2) .{ }^{\mathbf{6 2}, 63}$ To get insight into the mechanism of excited-state deactivation process, the minimum-energy reaction path (MEP) along the photophysically relevant reaction coordinates in the lowest excited singlet state were also determined with the ADC(2) method. For a suitably chosen driving coordinate, all other nuclear degrees of freedom were optimized 
for a given value of the driving coordinate. To allow costeffective exploration of the high-dimensional potential-energy surfaces, the standard split-valence double-zeta basis set with polarization functions on the heavy atoms (def-SV(P)) was employed in these geometry optimizations.

$A b$ initio calculations for all of the model systems were performed with the TURBOMOLE program package, ${ }^{53}$ making use of the Resolution-of-the-Identity (RI) approximation for the evaluation of the electron-repulsion integrals. ${ }^{64}$

\section{Acknowledgements}

This work was supported by the Polish National Science Centre (NCN), within the DEC-2013/09/B/ST4/00273 project. Laser flash photolysis studies were performed at the Center for Ultrafast Laser Spectroscopy at A. Mickiewicz University in Poznan. Gaussian 09 suite of programs were used at the PL-Grid Infrastructure.

\section{References}

1 D. Strack, T. Vogt and W. Schliemann, Phytochemistry, 2003, 62, 247-269.

2 M. Wendel, D. Szot, K. Starzak, D. Tuwalska, D. Prukala, T. Pedzinski, M. Sikorski, S. Wybraniec and G. Burdzinski, Dyes Pigm., 2015, 113, 634-639.

3 M. Wendel, D. Szot, K. Starzak, D. Tuwalska, J. Gapinski, R. Naskrecki, D. Prukala, M. Sikorski, S. Wybraniec and G. Burdzinski, J. Lumin., 2015, 167, 289-295.

4 F. Gandía-Herrero, F. García-Carmona and J. Escribano, J. Chromatogr. A, 2005, 1078, 83-89.

5 F. C. Stintzing, F. Kugler, R. Carle and J. Conrad, Helv. Chim. Acta, 2006, 89, 1008-1016.

6 F. Gandía-Herrero, F. García-Carmona and J. Escribano, Phytochem. Anal., 2006, 17, 262-269.

7 Y. Cai, M. Sun, W. Schliemann and H. Corke, J. Agric. Food Chem., 2001, 49, 4429-4435.

8 K. M. Herbach, F. C. Stintzing and R. Carle, J. Food Sci., 2006, 71, R41.

9 M. Piattelli, L. Minale and R. A. Nicolaus, Phytochemistry, 1965, 4, 817-823.

10 G. F. Trezzini and J. P. Zryd, Phytochemistry, 1991, 30, 19011903.

11 W. Schliemann, Y. Cai, T. Degenkolb, J. Schmidt and H. Corke, Phytochemistry, 2001, 58, 159-165.

12 F. Gandía-Herrero, F. Garcia-Carmona and J. Escribano, Food Res. Int., 2005, 38, 879-884.

13 F. Gandía-Herrero, M. Jiménez-Atiénzar, J. Cabanes, J. Escribano and F. García-Carmona, J. Agric. Food Chem., 2009, 57, 2523-2528.

14 F. Gandía-Herrero, F. García-Carmona and J. Escribano, Nature, 2005, 437, 334.

15 F. Gandía-Herrero, J. Escribano and F. Garcia-Carmona, Planta, 2010, 232, 449-460.

16 G. Calogero, J.-H. Yum, A. Sinopoli, G. D. Marco, M. Grätzel and M. K. Nazeeruddin, Sol. Energy, 2012, 86, 1563-1575.
17 D. Magde, R. Wong and P. G. Seybold, Photochem. Photobiol., 2002, 75, 327-334.

18 S. J. Strickler and R. A. Berg, J. Chem. Phys., 1962, 37, 814822.

19 S. A. Kovalenko, R. Schanz, V. M. Farztdinov, H. Hennig and N. P. Ernsting, Chem. Phys. Lett., 2000, 323, 312-322.

20 M. Wendel, S. Nizinski, D. Prukala, M. Sikorski, S. Wybraniec and G. Burdzinski, J. Photochem. Photobiol., A, 2017, 332, 602-610.

21 M. Wendel, S. Nizinski, D. Tuwalska, K. Starzak, D. Szot, D. Prukala, M. Sikorski, S. Wybraniec and G. Burdzinski, Phys. Chem. Chem. Phys., 2015, 17, 18152-18158.

22 G. R. Fleming, S. H. Courtney and M. W. Balk, J. Stat. Phys., 1986, 42, 83-104.

23 B. D. Allen, A. C. Benniston, A. Harriman, S. A. Rostron and C. Yu, Phys. Chem. Chem. Phys., 2005, 7, 3035-3040.

24 C. Singh, B. Modak, J. A. Mondal and D. K. Palit, J. Phys. Chem. A, 2011, 115, 8183-8196.

25 V. I. Stsiapura, A. A. Maskevich, V. A. Kuzmitsky, V. N. Uversky, I. M. Kuznetsova and K. K. Turoverov, J. Phys. Chem. B, 2008, 112, 15893-15902.

26 A. K. Singh, G. Ramakrishna, H. N. Ghosh and D. K. Palit, J. Phys. Chem. A, 2004, 108, 2583-2597.

27 W. P. Ozimiński and J. C. Dobrowolski, J. Phys. Org. Chem., 2009, 22, 769-778.

28 C. I. Oprea, A. Dumbrava, I. Enache, A. Georgescu and M. A. Girtu, J. Photochem. Photobiol., A, 2012, 240, 5-13.

29 S. S. Leang, F. Zahariev and M. S. Gordon, J. Chem. Phys., 2012, 136, 104101.

30 M. F. Rode and A. L. Sobolewski, Chem. Phys., 2008, 347, 413-421.

31 W. Domcke, D. R. Yarkony and H. Koppel, Conical Intersections: Theory, Computation and Experiment, World Scientific, 2011.

32 A. L. Sobolewski, W. Domcke and C. Hättig, J. Phys. Chem. A, 2006, 110, 6301-6306.

33 R. Omidyan and M. Iravani, J. Phys. Chem. A, 2016, 120, 1012-1019.

34 A. L. Sobolewski and W. Domcke, Phys. Chem. Chem. Phys., 2006, 8, 3410-3417.

35 R. H. E. Halabieh, O. Mermut and C. J. Barrett, Pure Appl. Chem., 2004, 76, 1445-1465.

36 P. Gorostiza and E. Y. Isacoff, Science, 2008, 322, 395-399.

37 G. Cui, P.-J. Guan and W.-H. Fang, J. Phys. Chem. A, 2014, 118, 4732-4739.

38 P.-J. Guan, G. Cui and Q. Fang, ChemPhysChem, 2015, 16, 805-811.

39 S. Kitzig, M. Thilemann, T. Cordes and K. Rueck-Braun, ChemPhysChem, 2016, 17, 1252-1263.

40 A. Szukalski, A. Miniewicz, K. Haupa, B. Przybyl, J. Janczak, A. L. Sobolewski and J. Mysliwiec, J. Phys. Chem. C, 2016, 120, 14813-14819.

41 A. Yartsev, J.-L. Alvarez, U. Aberg and V. Sundstrom, Chem. Phys. Lett., 1995, 243, 281-289.

42 B. Dietzek, A. Yartzev and A. N. Tarnovsky, J. Phys. Chem. B, 2007, 111, 4520-4526. 
43 Z. Wei, T. Nakamura, S. Takeuchi and T. Tahara, J. Am. Chem. Soc., 2011, 133, 8205-8210.

44 M. F. Rode and A. L. Sobolewski, Chem. Phys., 2012, 409, 4148.

45 M. F. Rode and A. L. Sobolewski, J. Chem. Phys., 2014, 140, 84301-84314.

46 L. Lapinski, M. J. Nowak, J. Nowacki, M. F. Rode and A. L. Sobolewski, ChemPhysChem, 2009, 10, 2290-2295.

47 M. F. Rode and A. L. Sobolewski, J. Phys. Chem. A, 2010, 114, 11879-11889.

48 J. Bahrenburg, M. F. Rode, A. L. Sobolewski and F. Temps, presented in part at the 19th International Conference on Ultrafast Phenomena, Okinawa Japan, 2014.

49 J. Jankowska, M. F. Rode, J. Sadlej and A. L. Sobolewski, ChemPhysChem, 2014, 15, 1643-1652.

50 S. Wybraniec, K. Starzak, A. Sipinska, B. Nemzer, Z. Pietrzykowski and T. Michalowski, J. Agric. Food Chem., 2013, 61, 6435-6476.

51 C. T. Lee, W. T. Yang and R. G. Parr, Phys. Rev. B: Condens. Matter Mater. Phys., 1988, 37, 785-789.

52 T. H. Dunning, J. Chem. Phys., 1989, 90, 1007-1023.

53 Development of University of Karlsruhe and Forschungszentrum Karlsruhe GmbH, TURBOMOLE GmbH, since 2007, http://www.turbomole.com, V6.3 2011 edn, 1989-2007.
54 A. Klamt and G. Schuumann, J. Chem. Soc., Perkin Trans. 1, 1993, 2, 799-805.

55 R. Krishnan, J. S. Binkley, R. Seeger and J. A. Pople, J. Chem. Phys., 1980, 72, 650.

56 S. Miertus, E. Scrocco and J. Tomasi, Chem. Phys., 1981, 55, 117.

57 R. Cammi, S. Corni, B. Mennucci and J. Tomasi, J. Chem. Phys., 2005, 122, 104513.

58 M. J. Frisch, G. W. Trucks, H. B. Schlegel, G. E. Scuseria, M. A. Robb, J. R. Cheeseman, G. Scalmani, V. Barone, B. Mennucci and G. A. Petersson, et al., in Gaussian 09, revision D.01, Gaussian, Inc., Wallingford, CT, USA, 2009.

59 S. N. Steinmann, C. Piemontesi, A. Delacht and C. Corminboeuf, J. Chem. Theory Comput., 2012, 8, 16291640.

60 A. Wakai, H. Fukasawa, C. Yang, T. Mori and Y. Inoue, J. Am. Chem. Soc., 2012, 134, 4990-4997.

61 C. Møller and M. S. Plesset, Phys. Rev., 1934, 46, 618-622.

62 J. Schirmer, Phys. Rev. A, 1981, 26, 2395.

63 A. B. Trofimov and J. Schirmer, J. Phys. B: At., Mol. Opt. Phys., 1995, 28, 2299.

64 F. Weigend and M. Häser, Theor. Chem. Acc., 1997, 97, 331340.

65 Y. Marcus, The Properties of Solvents, John Wiley \& Sons, Chichester, UK, 1998. 